\title{
Locating the Baryon Acoustic Peak
}

\author{
Fergus Simpson,, , * John A. Peacock, ${ }^{1}$ and Patrick Simon ${ }^{1}$ \\ ${ }^{1}$ Institute for Astronomy, University of Edinburgh, \\ Royal Observatory, Blackford Hill, Edinburgh EH9 $3 \mathrm{HJ}$
}

(Dated: November 4, 2018)

\begin{abstract}
Forthcoming photometric redshift surveys should provide an accurate probe of the acoustic peak in the two-point galaxy correlation function, in the form of angular clustering of galaxies within a given shell in redshift space. We investigate the form of the anticipated signal, quantifying the distortions that arise due to projection effects, and in particular explore the validity of applying the Limber approximation. A single-integral prescription is presented, which provides an alternative to Limber's equation, and produces a significantly improved prediction in the regime of interest.

The position of the acoustic peak within the angular correlation function relates to the angular diameter distance to the far side of the redshift bin. Thicker redshift bins therefore shift comoving features towards smaller angular scales. As a result, the value of the photometric redshift error acquires a greater significance, particularly at lower redshifts. In order to recover the dark energy equation of state to a level of $1 \%$, we find the total redshift dispersion must be determined to within $\Delta \sigma_{z} \lesssim 10^{-3}$, which may prove challenging to achieve in practice.
\end{abstract}

\section{INTRODUCTION}

The propagation distance of sound waves prior to decoupling is a convenient reference scale embedded in the distribution of galaxies. Lately these Baryon Acoustic Oscillations (BAO) have received particular attention due to their potential to measure the dark energy equation of state, $w[1,2,23,4]$. To attain competitive constraints on $w$, the angular scale of the BAO must be determined at the sub-percent level. In addition, robust models are required for any phenomena capable of inducing systematics of a comparable magnitude. In the near future, large photometric redshift surveys such as Pan-STARRS [15] and DES [16] will cover a substantial proportion of the sky, providing a vast map of large scale structure in the universe. However, the precision and reliability of the photometric redshifts will significantly influence the dark energy constraints. With a typical rms redshift error of $\sigma_{z} \sim 0.05$ the longitudinal modes of the power spectrum are exponentially damped, leaving only those which are closely aligned to the plane of the sky. Therefore BAOs from photometric redshift surveys are predominantly sensitive to the angular diameter distance $D_{A}$. This may also be interpreted as an effective reduction in volume given by approximately $12\left(\sigma_{z} /(1+z) / 0.03\right)$ [5], although Cai et al. [6] reach a more optimistic conclusion, suggesting only a fivefold reduction in volume.

The characteristic signature for a particular dark energy model resides in the apparent redshift variation of the angular acoustic scale. In order to study this behaviour, the galaxy population may be divided into thin redshift slices, limited in thickness to be $\gtrsim \sigma_{z}$. We explore the smoothing effect which arises when projecting from three dimensions down to two. Locating the acous-

*Electronic address: frgs@roe.ac.uk tic signature in Fourier space and real space - via the power spectrum and correlation function - are each susceptible to different forms of systematic errors. Finding both in agreement would therefore strengthen our confidence in any subsequent constraints on cosmological parameters. For this study we focus on the acoustic peak in the angular correlation function, paying particular attention to the influence of projection effects, and analyse the shift in peak position as a function of bin width. The Limber approximation, recently examined by Simon [7], acts as a starting point from which we develop an alternative analytic description of the transformation from the spatial to angular correlation function. Recently LoVerde \& Afshordi [8] express the angular power spectrum as a power series, within which the Limber approximation appears as the $0^{\text {th }}$ order term. Our approach is somewhat different, and we obtain a single-integral approximation for the angular correlation function, which provides a good description for galaxy redshift distributions of practical interest.

\section{FIDUCIAL COSMOLOGY}

To evaluate the spatial correlation function we begin with the linear matter power spectrum output from CAMB [9], and adopt the default cosmological parameters $\Omega_{m}=0.27 ; \Omega_{\Lambda}=0.73 ; \Omega_{b}=0.046 ; n_{s}=0.96 ; \sigma_{8}=$ 0.79. Throughout this work we assume a flat universe.

Conventional means of modelling non-linear structure formation, such as the HALOFIT prescription [10] are not well suited to an oscillatory $P(k)$ since they focus on corrections in the highly non-linear regime, rather than the quasilinear region where the BAO signal resides. Recently Eisenstein et al. [3] offered a simple approach to modelling the degradation of the acoustic peak. By considering the rms radial displacement for a pair of galaxies separated by $100 h^{-1} \mathrm{Mpc}$, the damping is given by 

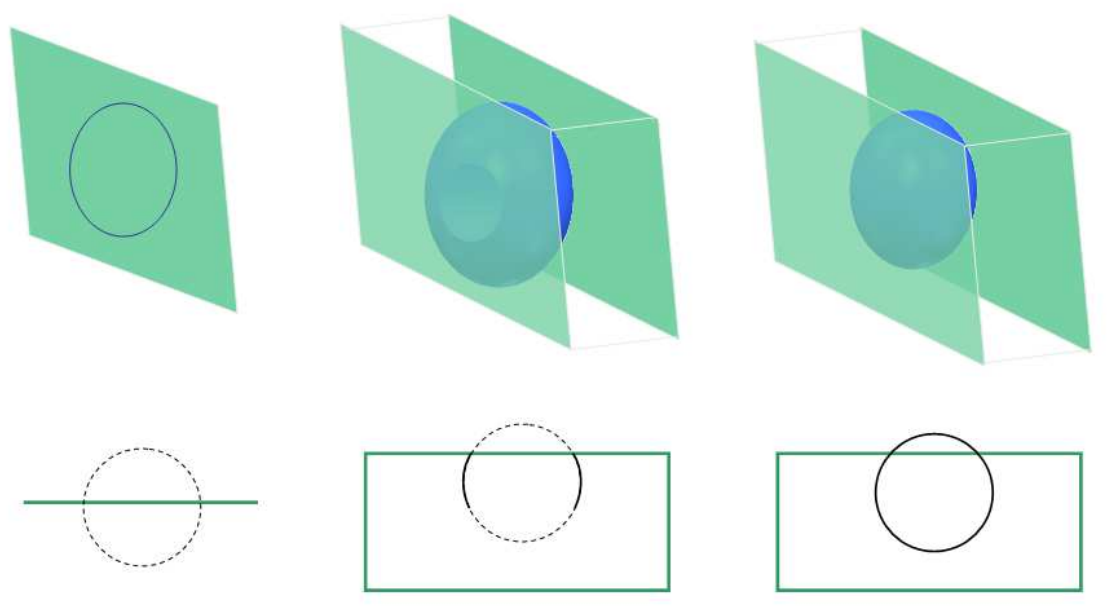

FIG. 1: A representation of the observable pairings in the case of (i) a thin redshift slice (ii) a thick redshift slice with truncation, as described in (IVB) and (iii) a thick redshift slice without truncation, as provided by the Limber approximation (8). Each point on the shell's surface - along with its corresponding antipode - represents a potential galaxy pair.

$$
\begin{gathered}
P(k)=P_{\text {linear }}(k) e^{-\frac{k^{2} \sigma^{2}}{2}}, \\
\sigma=s_{0} D,
\end{gathered}
$$

where $D \propto \delta(z) / \delta(0)$ is the growth function (normalised such that $D=a$ at early times), and $s_{0}$ is taken to be $10.9 h^{-1} \mathrm{Mpc}$, rescaled from the $12.4 h^{-1} \mathrm{Mpc}$ quoted in [3] to match our lower value of $\sigma_{8}$. The real-space correlation function at a given redshift is then obtained by

$$
\xi(r)=\frac{1}{2 \pi^{2}} \int P(k) \frac{k}{r} \sin (k r) \mathrm{d} k .
$$

However in redshift space the power is anisotropic, so before proceeding further we first explore the extent to which redshift distortions may impact our analysis. The anisotropic correlation function $\xi\left(r_{\perp}, r_{\|}\right)$, where $r_{\perp}$ and $r_{\|}$are the transverse and radial parts of $\underline{r}$, is related to the power spectrum via

$$
\begin{aligned}
& \xi\left(r_{\perp}, r_{\|}\right)=\frac{1}{(2 \pi)^{3}} \int P(\underline{k}) e^{i \underline{k} \cdot \underline{r}} \mathrm{~d}^{3} k \\
& =\frac{1}{(2 \pi)^{2}} \int_{-1}^{1} \int_{0}^{\infty} P(k, \mu) J_{0}\left(k r_{\perp} \sqrt{1-\mu^{2}}\right) e^{i \mu k r_{\|}} k^{2} \mathrm{~d} k \mathrm{~d} \mu
\end{aligned}
$$

where $\mu k$ is the radial part of $\underline{k}$. Thus e.g. $\xi\left(r_{\perp}, 0\right)$ is not in general the same as the real-space correlation function. Incorporating a simple model of the Kaiser and Fingers of God effects leaves us with

$$
\begin{aligned}
\xi\left(r_{\perp}, 0\right)= & \frac{1}{(2 \pi)^{2}} \int_{-1}^{1} \int_{0}^{\infty} P(k) J_{0}\left(k r_{\perp} \sqrt{1-\mu^{2}}\right) \times \\
& \left(1+\beta \mu^{2}\right)^{2} e^{-\mu^{2} k^{2}\left(\sigma_{z}^{2}+\sigma_{v}^{2}\right)} k^{2} \mathrm{~d} k \mathrm{~d} \mu
\end{aligned}
$$

where $\sigma_{v}$ denotes the one-dimensional rms single-point velocity dispersion. The strong damping of radial modes ensures that for all but the smallest values of $k$, contributions from the Kaiser effect are restricted to small values of $\mu$. This resembles a slow-varying scale-dependent bias in the angle-averaged power spectrum, which is therefore unproblematic provided we adopt an empirical "wiggles only" fitting function.

In practice we will want to consider angular clustering in shells of width comparable to the photo-z error, $\sigma_{z} \simeq 0.05$. This scale is large by comparison with those of peculiar velocities, so we shall ignore the above complication and treat the situation as though photo-z's are a noisy measure of the true radius, even though this is not strictly true. We shall thus tend to use the term 'redshift shell' to mean 'radial shell'.

\section{PROJECTION EFFECTS}

To begin, we consider an infinitesimally thin radial shell, so clustering may only be viewed perpendicular to the line of sight. In this case the angular correlation function simply resembles a rescaled version of the spatial correlation function, $w_{t}(\theta) \simeq \xi\left(\chi_{s} \theta\right)$, where $\chi_{s}$ is the (comoving) radial distance to the bin. This is known as the thin-layer approximation. Any given part of the spatial correlation function could therefore be represented as a circle on the sky, as illustrated in the left hand panel of Fig. 1.

When considering a thicker redshift bin, with a uniform selection function, two distinct phenomena influence our observation. The first, as represented by a truncated sphere in the centre panel of Fig. 1, is that galaxy pairs with significant line-of-sight displacements begin to contribute to the signal. Therefore any given large-scale 

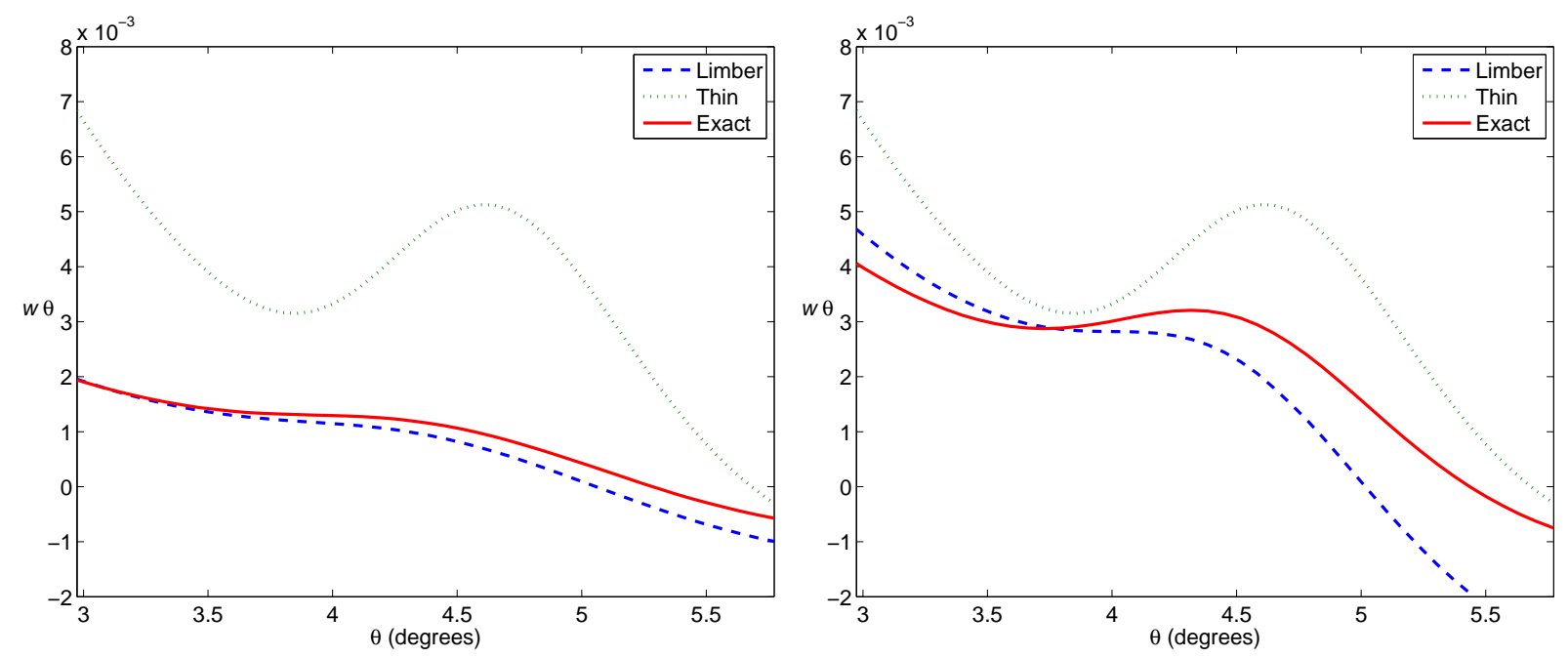

FIG. 2: The angular correlation function (solid) for a top-hat galaxy redshift distribution evaluated at $z=0.5$, compared to the Limber approximation (dashed), and thin-layer approximation (dotted). The bin widths are scaled such that $\Delta z=0.1(1+z)$ (left) and $\Delta z=0.04(1+z)$ (right).
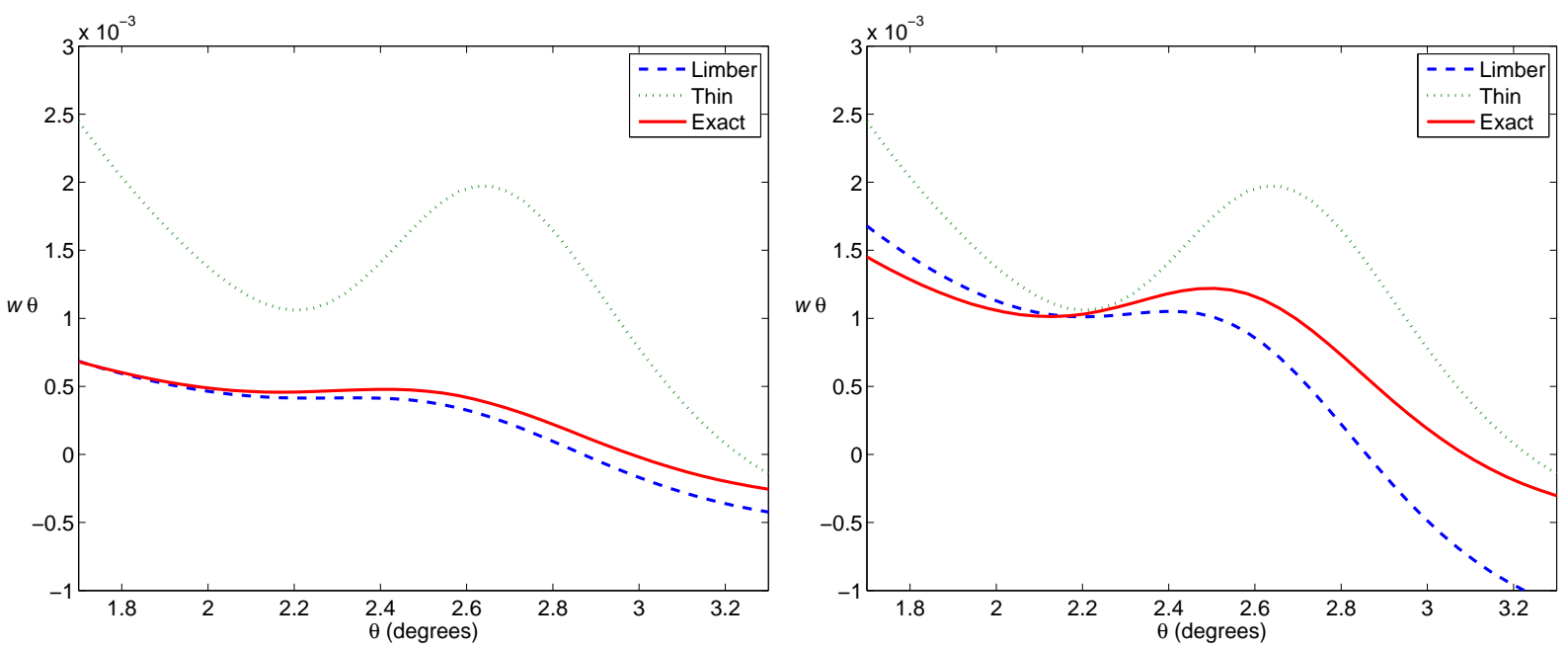

FIG. 3: The same plots as in Fig 2, but with the bin located at $z=1$.
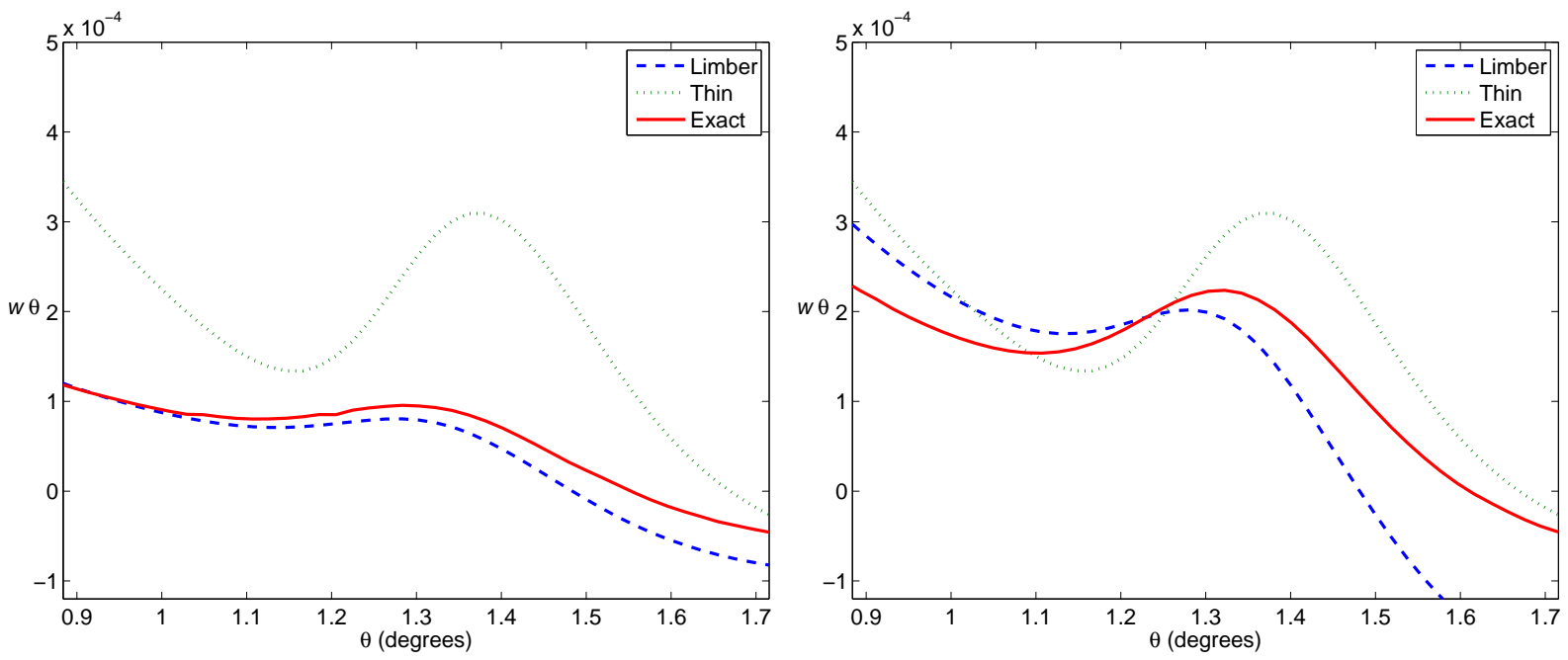

FIG. 4: The same plots as in Fig[2, but with the bin located at $z=3$. 
feature from $\xi(r)$ begins to influence the smaller angular scales of $w(\theta)$. The extent of this is governed by the ratio of the clustering scale to the bin thickness. The second effect arises since the truncated sphere subtends a different angle depending on its location within the bin. This effect gains prominence when the bin thickness is a significant proportion of the radial distance to the bin. This can often be alleviated by adopting a projected correlation function rather than an angular correlation function, but the presence of photometric redshift error proves to be a limiting factor.

For the case of a redshift bin much thicker than the clustering scale, as seen in the right hand panel of Fig. 1, our model is simplified since any truncation can be neglected and only the projection effect persists. Therefore any galaxy pair whose centre lies within the bin is assumed to contribute to the signal. This simplification leads to the erroneous inclusion of pairs which extend beyond the edge of the bin. Indeed this is the situation described by the Limber approximation, which provides us with a good starting point for the purposes of studying the acoustic peak. The clustering scale of interest is around $150 \mathrm{Mpc}$, whilst photometric redshift precision ensures the effective bin thickness is typically double this.

\section{A. Limber Shift}

The angular correlation function is evaluated numerically using (23) from Simon [7]

$$
w(\theta)=\left.\left.\frac{2}{1+\cos \theta} \iint \frac{\mathrm{d} n}{\mathrm{~d} y}\right|_{y-\frac{x}{2}} \frac{\mathrm{d} n}{\mathrm{~d} y}\right|_{y+\frac{x}{2}} \xi(y, R) \frac{R}{x} \mathrm{~d} R \mathrm{~d} y,
$$

where the variables $R, y$, and $x$ denote the galaxy pair separation, their mean distance from the observer, and their line-of-sight separation, as given by

$$
x \equiv \sqrt{\frac{2 R^{2}-4 y^{2}(1-\cos \theta)}{1+\cos \theta}},
$$

The evolution of $\xi$ is represented by its first argument, whilst the second provides the galaxy pair separation $R$. This formulation assumes that only linear evolution of $\xi$ occurs over the distance $x$, and no significant change in position of the nearer galaxy arises during the light-travel time between the pair.

Projection effects lead to the acoustic peak appearing at a smaller angular scale than one might naïvely expect. Here we assess whether a simplified form of (6) may be used to provide an estimate of the acoustic peak location. The Limber approximation is given by

$$
w(\theta)=\int_{0}^{\infty} \int_{-\infty}^{\infty}\left(\frac{\mathrm{d} n}{\mathrm{~d} y}\right)^{2} \xi\left(y, \sqrt{x^{2}+y^{2} \theta^{2}}\right) \mathrm{d} x \mathrm{~d} y
$$

This simplification utilises the flat-sky approximation, which holds well even on the relatively large angular scales under consideration. At a separation of $5^{\circ}$ the approximation $(1+\cos \theta) \sim 2$ provides an overestimation of $0.2 \%$, and this propagates to $R$ with an error of less than $0.1 \%$. This is more prominent for the longitudinal modes, to which we are least sensitive.

Figs. 2, 4 illustrate the shift in the acoustic peak as predicted by the Limber equation. Note how the acoustic peak is almost entirely erased when the bin width is $\Delta z=0.1(1+z)$, highlighting the need for accurate photometric redshifts. The increasing bin thickness, chosen to mimic the degradation of the photometric redshifts, is counteracted by the reduced value of $\mathrm{d} \chi / \mathrm{d} z$ at higher redshift, leading to little change in the projection effect across the range of redshifts $0.5<z<3$. Ordinarily the Limber approximation overestimates the signal, however an underestimate arises here due to the influence of the correlation function's negative regime. The Limber approximation clearly offers a more appropriate estimation than that of the thin layer. Its precision varies according to the nature of the chosen fitting algorithm, and improves with broader bins. We find the relevant angular scale is underestimated, with the magnitude of the discrepancy typically ranging from $2 \%$ to $5 \%$, substantially exceeding the level of accuracy required for dark energy studies.

\section{SHIFT ESTIMATION}

As we have seen, adopting the Limber approximation results in a good but insufficiently accurate estimation for the positioning of the acoustic peak. Here we attempt to gain a quantitative understanding of the shift, and interpret this as a smearing of $w_{t}(\theta)$, the correlation function from an infinitesimal bin.

\section{A. Limber Equation}

We begin by transforming the Limber equation to plane polar coordinates

$$
w(\theta)=\frac{2}{\theta} \int_{0}^{\pi / 2} \int_{0}^{\infty}\left(\frac{\mathrm{d} n}{\mathrm{~d} y}\right)^{2} \xi(r) r \mathrm{~d} r \mathrm{~d} \theta^{\prime}
$$

where we substitute $x=r \sin \theta^{\prime}, y \theta=r \cos \theta^{\prime}$.

For the moment we neglect the redshift evolution in the correlation function, and assume a top-hat galaxy distribution, simplifying our equation by assuring $\mathrm{d} n / \mathrm{d} y=$ $(\Delta \chi)^{-1}$ within the top-hat, where $\Delta \chi$ corresponds to the physical depth of the redshift bin. Later we shall see that our results are largely insensitive to the functional form of the redshift bin, and the impact of an evolving $\xi(\chi)$ across reasonably narrow redshift bins is minimal.

These simplifications leave us with an angular correlation function given by 


$$
w(\theta)=\frac{2}{\theta \Delta \chi^{2}} \int_{\theta_{1}^{\prime}}^{\theta_{2}^{\prime}} \int_{0}^{\infty} \xi(r) r \mathrm{~d} r \mathrm{~d} \theta^{\prime},
$$

and it is instructive to study the case where the correlation function consists of a delta function at an arbitrary location $r_{0}$ :

$$
\begin{gathered}
\xi(r)=\delta\left(r_{0}-r\right), \\
w(\theta)=\frac{2 r_{0}}{\theta \Delta \chi^{2}} \int_{\theta_{1}^{\prime}}^{\theta_{2}^{\prime}} \mathrm{d} \theta^{\prime},
\end{gathered}
$$

where the lower integration limit is given by

$$
\begin{gathered}
\theta_{1}^{\prime}=\arccos \left(\frac{\chi_{1} \theta}{r_{0}}\right), \\
\chi_{1}=\chi_{s}+\frac{\Delta \chi}{2},
\end{gathered}
$$

and similarly for $\theta_{2}^{\prime}$ :

$$
\begin{gathered}
\theta_{2}^{\prime}=\arccos \left(\frac{\chi_{2} \theta}{r_{0}}\right), \\
\chi_{2}=\chi_{s}-\frac{\Delta \chi}{2},
\end{gathered}
$$

where we denote the upper and lower boundaries of the galaxy distribution by $\chi_{1}$ and $\chi_{2}$ respectively, and define $\arccos (x)=0$ for $x>1$. This leaves us with

$$
w_{s}(\theta)=\frac{2 r_{0}}{\theta \Delta \chi^{2}}\left[\arccos \left(\frac{\chi_{2} \theta}{r_{0}}\right)-\arccos \left(\frac{\chi_{1} \theta}{r_{0}}\right)\right] .
$$

Substituting $r_{0}=\chi_{s} \theta_{0}$ leaves us with the Limber smear function

$$
w_{s}(\theta)=\frac{2 \chi_{s}^{2} \theta_{0}}{\theta \Delta \chi^{2}}\left[\arccos \left(\frac{\chi_{2} \theta}{\chi_{s} \theta_{0}}\right)-\arccos \left(\frac{\chi_{1} \theta}{\chi_{s} \theta_{0}}\right)\right] .
$$

The functional form of this is illustrated in Fig. 5, and this highlights the mechanism by which the peak position is shifted to smaller angular scales, typically by around $10 \%$. The projected signal peaks at $\theta_{p}$, the angle corresponding to the far edge of the redshift bin, as given by

$$
\theta_{p}=\frac{\chi_{s}}{\chi_{1}} \theta_{0}
$$

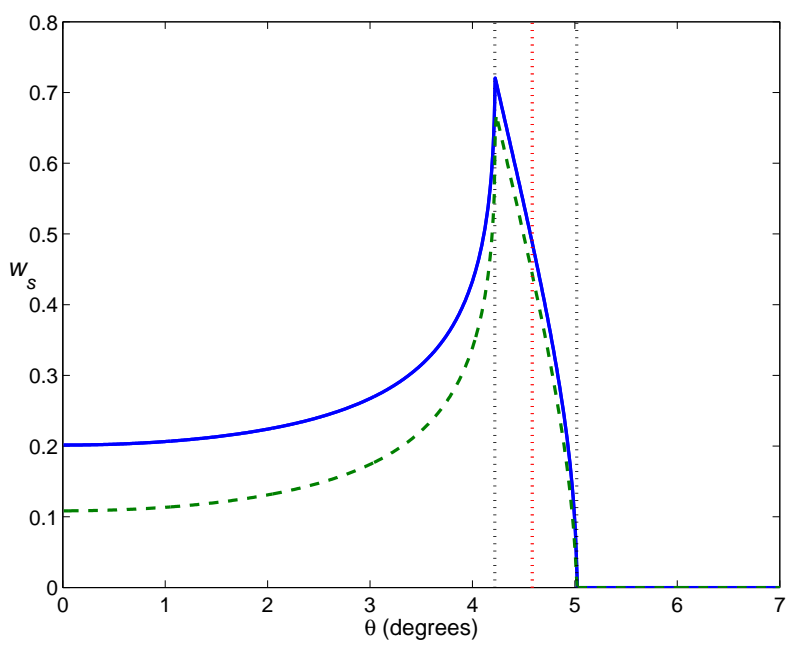

FIG. 5: The solid line represents the smear function derived from the Limber approximation, $w_{s}$ as defined in (18), and evaluated at $z=0.5$ with $\Delta z=0.1$. The dashed line is the exact expression, illustrating the modification introduced by accounting for the finite bin width. On smaller angular scales, corresponding to a large line-of-sight separation, the pairs lost from truncation become particularly apparent. Of the three vertical dotted lines, the central one represents the original $\delta$-function, while the left and right lines denote the angular scales associated with the far and near bin edges respectively.

At greater angular scales contributions are rapidly lost, starting from those at the far edge of the bin, with the signal reaching zero at the angular scale corresponding to the near edge of the bin. Conversely, smaller angular scales gradually receive fewer contributions since they require the pair orientation to be increasingly aligned with the line of sight. These are the pairs which prove particularly susceptible to overestimation by the Limber equation, since they readily protrude beyond the bin boundary.

Now returning to (18), and extending our analysis to a general correlation function, the parameter $\theta_{0}$ becomes a variable over which we integrate

$$
w_{\mathrm{L}}(\theta)=\int_{0}^{\infty} w_{s}\left(\theta, \theta_{0}\right) \xi\left(\chi_{s} \theta_{0}\right) \mathrm{d} \theta_{0} .
$$

\section{B. Correcting for Bin Width}

Here we modify the limits of our integration in order to account for the finite bin width. This aims to improve on the Limber approximation's estimate for the location of the acoustic peak.

Towards the bin edges the spherical shell assumption breaks down - a galaxy cannot pair up with another galaxy which lies beyond the bin boundary. This truncation is particularly problematic on scales which are large relative to the bin width (see Fig. 1). By considering 
the point at which one of the galaxy pair extends beyond the bin boundary, we shrink the integration range accordingly, with our new limits given by

$$
\theta_{1}^{\prime}=\arccos \left[\frac{\left(\chi_{1}-\delta \chi_{1}\right) \theta}{r_{0}}\right]
$$

where the distance from the bin edge $\delta \chi_{1}$ is

$$
\begin{gathered}
\delta \chi_{1}=\frac{\chi_{s} \theta_{0}}{2} \cos \left(\frac{\theta}{2}-\phi_{1}\right), \\
\sin \phi_{1}=\frac{\chi_{1} \theta}{\chi_{s} \theta_{0}} \cos \left(\frac{\theta}{2}\right),
\end{gathered}
$$

similarly

$$
\begin{gathered}
\theta_{2}^{\prime}=\arccos \left[\frac{\left(\chi_{2}+\delta \chi_{2}\right) \theta}{r_{0}}\right] \\
\delta \chi_{2}=\frac{\chi_{s} \theta_{0}}{2} \cos \left(\frac{\theta}{2}+\phi_{2}\right), \\
\sin \phi_{2}=\frac{\chi_{2} \theta}{\chi_{s} \theta_{0}} \cos \left(\frac{\theta}{2}\right) .
\end{gathered}
$$

The angular correlation function is then recovered by

$$
\begin{gathered}
w(\theta)=\int_{0}^{\infty} w_{c}\left(\theta, \theta_{0}\right) \xi\left(\chi_{s} \theta_{0}\right) \mathrm{d} \theta_{0}, \\
w_{c}\left(\theta, \theta_{0}\right)=\frac{2 \chi_{s}^{2} \theta_{0}}{\theta \Delta \chi^{2}}\left(\theta_{1}^{\prime}-\theta_{2}^{\prime}\right) .
\end{gathered}
$$

This expression is exact within the context of a uniform galaxy distribution and flat sky approximation.

The influence of this adjustment is illustrated by the dashed line in Fig 5 . The Limber equation generally overestimates the amplitude of the correlation function due to over-counting the number of pairs, and this truncated integral provides the necessary correction. Upon evaluating the integral, we recover the solid and dashed lines originally shown in Fig, 2 ,

\section{THE GALAXY DISTRIBUTION}

Up until now we have considered a uniform galaxy distribution. Provided $\Delta \chi / \chi$ is small, the functional form of $n(z)$ has a limited impact on the appearance of the correlation function. We find that the $w(\theta)$ produced by a Gaussian selection function may be closely mimicked

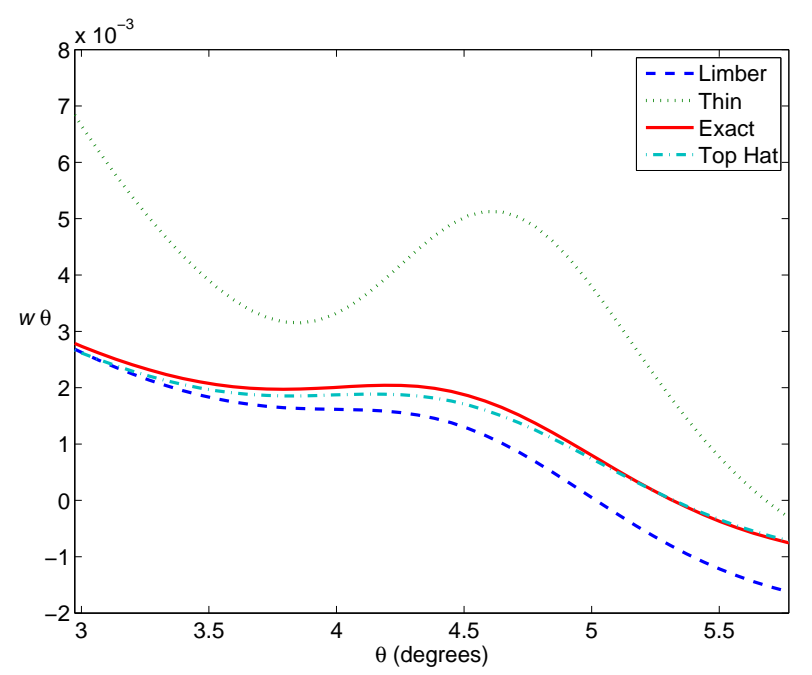

FIG. 6: Here we take the angular correlation function corresponding to a Gaussian redshift distribution at $z=0.5$ with $\sigma_{z}=0.02(1+z)$, and superpose that of a uniform galaxy distribution (dot-dash), scaled to provide a matching rms.

by simply selecting a top-hat selection function, of width $\Delta z$ chosen with a matching rms, given by

$$
\Delta z=\sqrt{12} \sigma(n(z))
$$

Fig. 6 compares three estimates of the angular correlation function for a Gaussian distribution. We find that this top-hat approximation compares favourably to the conventional Limber and thin layer approaches, producing a more faithful reproduction of the bump's shape and magnitude. Yet for higher precision and larger values of $\Delta z / z$, inevitably one must return to the exact form given by (6).

\section{CALIBRATION REQUIREMENTS}

In this section we clarify what constraints on the photometric redshift errors are needed in order to avoid significant degradation or biasing of the dark energy constraints from future surveys.

\section{A. Photometric Redshift Error}

When projecting from the $3 \mathrm{D}$ correlation function to the two-dimensional angular correlation function, not only does the smoothing process dampen the appearance of the peak, but its angular position shifts in relation to the thickness of the redshift bin. This modified peak location, previously illustrated in Figure 5, is associated with the angular diameter distance at the far edge of the redshift bin. It is via this mechanism that the magnitude of the photometric redshift error controls the peak 


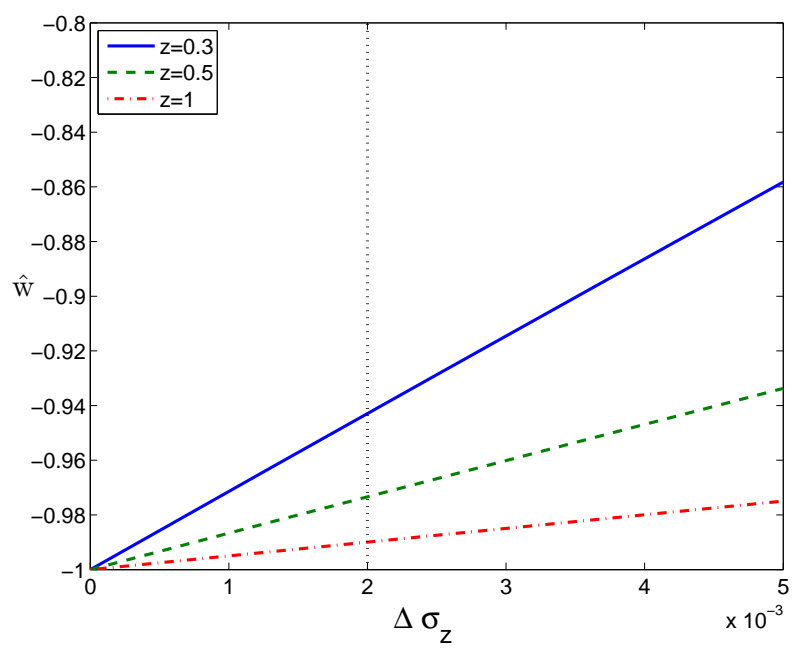

FIG. 7: Overestimating the photometric redshift error may lead to a substantial overestimate of the dark energy equation of state, particularly at lower redshifts. The vertical line denotes the achievable limit in the near-term future, as given by Newman [11].

position. Any uncertainty in $\sigma_{z}$ therefore influences our estimate of the dark energy equation of state.

To quantify this effect we select a top-hat of width $\Delta z_{p}=0.1$ in photometric redshift space, which is then convolved with a Gaussian of standard deviation $\sigma_{z}$ to simulate the true redshift distribution. As demonstrated in the previous section, the resulting angular correlation function is readily approximated by simply adopting a pure top-hat selection function of width

$$
\Delta z=\sqrt{\Delta z_{p}^{2}+12 \sigma_{z}^{2}}
$$

and the redshift of interest is $z_{1}$, the far edge of the bin

$$
z_{1}=\bar{z}+\frac{\Delta z}{2}
$$

It is therefore crucial to acquire accurate and unbiased estimates of both the mean redshift $\bar{z}$ and the photometric error $\sigma_{z}$. Note that from (31) we find

$$
\frac{\mathrm{d} z_{1}}{\mathrm{~d} \sigma_{z}}=\frac{6 \sigma_{z}}{\Delta z}
$$

thus if we take $\Delta z_{p}=2 \sigma_{z}$, this leaves our measurement $50 \%$ more sensitive to the value of $\sigma_{z}$ compared to the mean redshift $\bar{z}$. A selection function much wider than $2 \sigma_{z}$ is undesirable as it leads to excessive damping of the peak, and leaves us susceptible to the redshift evolution of $\sigma_{z}$.

To proceed, we assume the mean redshift is perfectly known, and explore the impact of $\sigma_{z}$ on our estimation of the dark energy equation of state.

\section{B. Dark Energy Constraints}

The process of fitting the acoustic peak to the data is not straightforward. A strict fitting regime corresponding to the anticipated shape of $\xi(r)$ from simulations would leave us vulnerable to systematic errors. Meanwhile the adoption of a fitting function with many degrees of freedom would substantially degrade the precision of our measurement. To avoid introducing any dependence on the fitting method into our results, we simply consider the angular shift induced on a correlation function of the form $\xi(r)=\delta\left(r_{0}-r\right)$, where $r_{0}=110 h^{-1} \mathrm{Mpc}$.

In Figure 7 we evaluate the impact of an uncertainty in $\sigma_{z}$ at redshifts $z=0.3,0.5,1$, with fixed bin width $\Delta z_{p}=0.1$ and $\sigma_{z}=0.05(1+z)$. We incorporate an error $\Delta \sigma_{z}$ defined such that

$$
\Delta z=\sqrt{\Delta z_{p}^{2}+12\left(\sigma_{z}+\Delta \sigma_{z}\right)^{2}},
$$

Our estimate of the equation of state, $\hat{w}$, is then perturbed by an amount relating to $\mathrm{d} D_{A} / \mathrm{d} z_{1}$, thus leading to the steeper slopes at lower redshift. Provided the estimator is unbiased, averaging over several bins could improve the situation. Conversely, bypassing the CMB by taking the ratios of angular scales in different bins would further aggravate the problem.

\section{Measuring $\sigma_{z}$}

The conventional method for estimating the photometric redshift error is to use a calibration set of spectroscopic redshifts covering a suitably representative sample of galaxies. Newman [11] provided an estimate of $\Delta \sigma_{z}$ attainable from spectroscopic calibration samples, combined with information from their angular clustering, and found $\Delta \sigma_{z} \sim 2 \times 10^{-3}$. This includes a modelling of the outlier population as a second Gaussian with a significantly larger standard deviation.

Zhan \& Knox [12] consider using the exponential damping of the longitudinal modes of the galaxy power spectrum to determine the ratio $\sigma_{z} / H$, where $H$ is the Hubble parameter at the redshift of interest. Applying a prior on $H$ will then provide an estimate of $\sigma_{z}$. For example, for an LSST-like survey, they find a 1\% prior on $H$ leads to an uncertainty on $\sigma_{z}$ of $1 \%$. Ma et al. [13] explore the impact of photometric redshift uncertainties on weak lensing surveys, which appear to have photometric calibration requirements at a similar level to that of the BAO.

The non-Gaussianity of photometric redshift errors, along with their strong variation with redshift (see Fig 8 of [6]), ensures that we require more degrees of freedom than simply $\sigma_{z}$, which further heightens the difficulty of reaching the required level of precision. Ma \& Bernstein 14] estimate this may be compensated for by enlarging the spectroscopic calibration sample by a factor of five. 


\section{THE PROJECTED CORRELATION FUNCTION}

Here we justify the choice of an angular correlation function, used throughout this work, as opposed to the projected correlation function. This is defined such that

$$
w_{p}\left(r_{\perp}\right) \equiv 2 \int_{r_{\perp}}^{\infty} \xi(r) \frac{r}{\sqrt{r^{2}-r_{\perp}^{2}}} \mathrm{~d} r .
$$

To evaluate this quantity we require the precise location of the galaxy in the bin, but this is unknown since $\sigma_{z} \sim \Delta z$. As a result, adopting this approach aggravates the smoothing of the correlation function. To illustrate this we consider the range of transverse separations which are observed by a particular value of $w(\theta)$ and $w_{p}\left(\theta \chi_{s}\right)$. For the angular correlation function this is simply given by the limits $\chi_{1} \theta$ and $\chi_{2} \theta$. However with the projected correlation function this window is extended to $\left(\theta \chi_{s} \chi_{1} / \chi_{2}\right)$ and $\left(\theta \chi_{s} \chi_{2} / \chi_{1}\right)$ respectively. This occurs when pairs thought to be located at $\chi_{1}$ are actually at $\chi_{2}$, and vice versa.

Conversely when studying a bin with $\sigma_{z}<\Delta z$ the projected correlation function is the more natural option, and will be subject to projection effects in a manner closely analogous to that considered here.

\section{DISCUSSION}

We present a single-integral solution to the angular correlation function, by considering the special case of a uniform galaxy distribution. This provides a useful alternative to the traditional Limber and thin-layer approximations, and offers a substantial improvement in accuracy when evaluating the appearance and location of the acoustic peak.

This top-hat approximation can be utilised to derive an analytic estimate for the impact of photometric redshift errors on BAO surveys. In agreement with Zhan \& Knox [12], we find the value of $\sigma_{z}$ ought to be known to better than $\sim 10^{-3}$ if the acoustic peak is to provide significant and robust constraints on the dark energy equation of state, and this is particularly apparent for lower redshifts, $z<1$. It also seems likely that the anticipated departure from Gaussianity of this error distribution must be well understood.

We have not addressed the complexities associated with galaxy bias, since the same projection process applies regardless of the original form of the spatial correlation function. However, within the context of a photometric redshift survey a well-defined model of $\xi(r)$ will be required in order to reverse the smoothing process.

Whilst future projects such as LSST and SNAP will look to higher redshifts, it is the near-term projects such as Pan-STARRS and DES which, despite their higher tolerance for statistical errors, may be more susceptible to the difficulties in mapping the low-redshift behaviour of $\sigma_{z}(z)$.

Acknowledgements

FS was funded by an STFC rolling grant, and is grateful for the generous welfare support from the University of Edinburgh. PS acknowledges support by the European DUEL Research-Training Network (MRTN-CT2006-036133).
[1] C. Blake and K. Glazebrook, Astrophys. J. 594, 665 (2003).

[2] H.-J. Seo and D. J. Eisenstein, Astrophys. J. 598, 720 (2003).

[3] D. J. Eisenstein, I. Zehavi, D. W. Hogg, R. Scoccimarro, M. R. Blanton, R. C. Nichol, R. Scranton, H.-J. Seo, M. Tegmark, Z. Zheng, et al., Astrophys. J. 633, 560 (2005).

[4] S. Cole, W. J. Percival, J. A. Peacock, P. Norberg, C. M. Baugh, C. S. Frenk, I. Baldry, J. Bland-Hawthorn, T. Bridges, R. Cannon, et al., Mon.Not.Roy.As.Soc. 362, 505 (2005).

[5] C. Blake and S. Bridle, Mon.Not.Roy.As.Soc. 363, 1329 (2005), arXiv:astro-ph/0411713.

[6] Y.-C. Cai, R. E. Angulo, C. M. Baugh, S. Cole, C. S. Frenk, and A. Jenkins, ArXiv e-prints (2008), 0810.2300.

[7] P. Simon, Astron. \& Astrophys. 473, 711 (2007), arXiv:astro-ph/0609165.
[8] M. LoVerde and N. Afshordi, ArXiv e-prints (2008), 0809.5112.

[9] A. Lewis, A. Challinor, and A. Lasenby, Astrophys. J. 538, 473 (2000), arXiv:astro-ph/9911177.

[10] R. E. Smith, J. A. Peacock, A. Jenkins, S. D. M. White, C. S. Frenk, F. R. Pearce, P. A. Thomas, G. Efstathiou, and H. M. P. Couchman, Mon.Not.Roy.As.Soc. 341, 1311 (2003), arXiv:astro-ph/0207664.

[11] J. A. Newman, Astrophys. J. 684, 88 (2008), 0805.1409.

[12] H. Zhan and L. Knox, Astrophys. J. 644, 663 (2006), arXiv:astro-ph/0509260.

[13] Z. Ma, W. Hu, and D. Huterer, Astrophys. J. 636, 21 (2006), arXiv:astro-ph/0506614.

[14] Z. Ma and G. Bernstein, Astrophys. J. 682, 39 (2008), 0712.1562 .

[15] http://pan-starrs.ifa.hawaii.edu/public/

[16] http://www.darkenergysurvey.org/ 